\title{
Implementation of Assertive Community Treatment in Australia: model fidelity, patient characteristics and staff experiences
}

Carol Harvey, Associate Professor, Department of Psychiatry, The University of Melbourne \& North Western Mental Health, Psychosocial Research Centre, 130 Bell Street, Coburg, Victoria 3058, Australia.

Helen Killaspy, Reader, Department of Mental Health Sciences, University College London and Camden and Islington NHS Foundation Trust, Royal Free Hospital, London NW3 2PF, United Kingdom.

Salvatore Martino, Psychiatrist, formerly South West Area Mental Health Service, Melbourne, Australia (currently 286 Taronga Rd, Glen Iris, Victoria 3146, Australia).

Sonia Johnson, Professor, Research Department of Mental Health Sciences, University College London and Camden and Islington NHS Foundation Trust, Charles Bell House, 67-73 Riding House Street, London W1W 7EY, United Kingdom.

\section{$\underline{\text { Address for correspondence: }}$}

Dr Carol Harvey

Associate Professor in Psychiatry

Psychosocial Research Centre

130 Bell St

Coburg

Victoria 3058

Phone: 61393550825

Fax: 61393559855

c.harvey@unimelb.edu.au 


\title{
Implementation of Assertive Community Treatment in Australia: model fidelity, patient characteristics and staff experiences
}

\begin{abstract}
The impact of variable implementation of the Assertive Community Treatment (ACT) model on patient outcomes is increasingly recognised. We conducted the first study of four established Australian ACT teams, examining team composition, processes and model fidelity, using previously validated questionnaires. Demographic and clinical details of patients and their own experiences of ACT were gathered from staff. Associations between burnout and work experiences were examined. All teams were ACT-like (mean DACTS score $=3.7, \mathrm{SD}=0.3$ ) with few significant patient differences between teams, except diagnosis (schizophrenia 61\% to 93\%, co-morbid substance abuse $16 \%$ to $33 \%$ ) and proportion living alone (23\% to $72 \%$ ). Clinicians were fairly satisfied, but inter-team differences in staffing profile and experience emerged and one team scored highly on emotional exhaustion. Increased burnout was associated with greater stress due to taking a team approach. Inter-team differences suggested that attention to effective team working and leadership, as well as model fidelity, may be warranted.
\end{abstract}

Keywords: Assertive community treatment; community mental health services; severe mental illness; schizophrenia; staff burnout; team working. 


\section{Introduction}

Assertive Community Treatment (ACT) originated from Stein and Test's experimental home-based treatment service (Stein \& Test, 1980) which provided an alternative to admission to psychiatric hospital. This gradually evolved into two specific models of community care, ACT (Marshall \& Lockwood, 1998; McGrew, Bond, Dietzen, \& Salyers, 1994) and crisis resolution (Joy, Adams, \& Rice, 2002). ACT is indicated for patients with serious mental illnesses who have problems engaging with services and have experienced frequent hospital admissions. Systematic reviews have concluded that, when targeted at high users of inpatient services, it reduces the costs of care by decreasing frequency and length of admissions (Marshall \& Lockwood, 1998).

There is strong evidence for ACT in the USA (Marshall \& Lockwood, 1998) where, in addition to decreased inpatient use, trials have reported improved contact between patients and services, improved patient satisfaction, and better employment and housing outcomes in comparisons with usual care. The only randomised controlled trial of ACT in Australia was a successful replication of the original Stein and Test (1980) study (Hoult, 1986). Subsequent Australian evaluations of ACT have shown significant reductions in the number of patients admitted to hospital and in hospital bed days, a significant increase in functioning and a significant decrease in non-psychotic symptoms, (Hambridge \& Rosen, 1994). Further, a randomised control comparison of an ACT-like intensive case management model reported that patients had improved social functioning, fewer hospital admissions involving police and were more likely to engage and remain in treatment (Issakidis, Sanderson, Teeson, Johnson, \& Buhrich, 1999).

The case for the efficacy of ACT in the UK and the rest of Europe has been less clear. Studies such as PRiSM (Thornicroft, Wykes, Holloway, Johnson, \& Szmukler, 1998) and UK700 (Burns et al., 1999) found no particular advantage for more intensive community mental health services over usual care from community mental health teams (CMHTs) although neither study was specifically designed to test the ACT model (Marshall et al., 1999). However, results from the REACT study (Killaspy et al., 2006), a randomised controlled trial of ACT versus usual care from CMHTs in inner London, appeared to concur with these earlier studies, finding no difference in bed days, symptoms or social function between the two groups. Overall, European findings suggest that ACT results in better engagement of patients and higher patient satisfaction than comparison care, without the other beneficial outcomes reported in the US and 
Australia (Sytema, Wunderink, Bloemers, Roorda, \& Wiersma, 2007). Various explanations have been advanced for this failure to discern an effect of ACT in some countries. ACT teams that were not established for long enough or followed up for a sufficient period to obtain significant outcomes, or with low fidelity to the original ACT model (Teague, Bond, \& Drake, 1998), may provide some explanation. More recent work has identified several "critical ingredients" of the model which may be important for effectiveness, namely, extended hours of operation, home visiting and the team approach (Catty et al., 2002; Burns et al., 2007). Further, a treatment effect would be unlikely to be detected if the comparison care was of a higher standard than in the original RCTs.

Although unlikely to provide the entire explanation for differences in the international evidence (Fiander, Burns, McHugo, \& Drake, 2003; King, 2006), model fidelity has assumed a particular importance in the ACT research for a number of reasons. High fidelity programs are more effective (McHugo et al., 2007) and fidelity explains much of the variation in client outcomes (Burns et al., 2007). Despite this, implementation of ACT programs may vary (Bond, 1990) and insufficient is known about what is good or adequate implementation (Salyers et al., 2003). For example, the Pan London Assertive Outreach (PLAO) studies (Billings et al., 2003; Priebe et al., 2003; Wright et al., 2003) showed great heterogeneity in implementation of ACT in London, with at least three types of ACT team and only four of the 24 teams achieving high fidelity to the ACT model (Teague et al., 1998; Wright et al., 2003). Staff experiences, especially burnout, are another important consideration in ACT implementation. Burnout has been associated with diminished collegial support, which is linked in turn with poor leadership and staff feeling bogged down with bureaucratic constraints and a lack of control (Corrigan et al, 2001). Conversely, cooperative interdisciplinary team work and support partially protects against burnout (Rosen et al, 2007). Encouragingly, most ACT staff in the PLAO studies were not experiencing high levels of burnout although teams were relatively new (Billings et al, 2001).

In the context of successful replication of the ACT research in Australia, the ACT model was adopted within some states from the early 1990s (Ash et al., 2007). In Victoria, a general framework for adult mental health services was introduced, drawing on the international evidence, which included crisis teams, ACT teams, CMHTs, acute inpatient units and long term residential care in the community (Health and Community Services, 1994). As a consequence, Mobile Support and Treatment Teams (MSTTs) based on 
the ACT model have been established for some time (Human Services Victoria, 1995). To our knowledge, there has been no previous Australian study of ACT model fidelity or staff experiences.

The aim of this study was to investigate four ACT teams in Melbourne, Victoria: (i) to provide the first description of the real-world implementation of ACT teams in Australia, including team organisation and service delivery, staff characteristics and experiences and patient characteristics; (ii) to investigate model fidelity (including critical ingredients of the model) since it could explain differences in outcomes for ACT reported in Europe and Australia; (iii) to investigate staff burnout in established ACT teams and their associations with work satisfaction and stress; and (iv) to provide a fine-grained comparison of the 4 teams, with a particular emphasis on critical model ingredients. Given the ongoing international debate about implementation and effectiveness, similar measures to those employed in the PLAO studies were used, so

as to enhance the understanding of cross-national differences. Detailed comparisons between these Melbourne teams and those investigated in the PLAO studies (Billings et al., 2003; Priebe et al., 2003; Wright et al., 2003) are reported elsewhere (Harvey et al, In press).

\section{Methods}

\section{Setting}

This study was approved by the Melbourne Health (MHREC 2003.019) and Werribee Mercy (R03/07W) Research and Ethics Committees. All authors declare that there are no known conflicts of interest and certify responsibility for this manuscript.

The study was conducted in four Area Mental Health Services (AMHS) located within two adjacent service administrations in Western Melbourne, between August 2003 and December 2004. Mental Health Services provided by each AMHS were consistent with the Framework paper (Health and Community Services, 1994), already described. A separate service provided psychiatric care for adolescents, including treatment for first episode psychosis, within the same geographical area. Under the Victorian Mental Health Act (1986) (Victorian Government, 1986) AMHS patients could be compulsorily treated under Community Treatment Orders (CTOs, legislative orders which compel outpatient treatment). 
The four AMHS vary in total population from 170K to 284K (Australian Bureau of Statistics, 2003a) and in size from 80 to 888 square kilometres. Each serves a culturally diverse population, including both migrants and refugees. The Australian Bureau of Statistic's Index of Relative Socio Economic Disadvantage (IRSED) is derived from Census variables selected to reflect disadvantage (Australian Bureau of Statistics, 2003b). Three of the catchment areas were ranked within the four most disadvantaged Victorian AMHSs; the fourth was the $14^{\text {th }}$ most disadvantaged of the 21 Victorian AMHSs.

\section{Participants}

Participants were all clinical staff of the four MSTTs. Data on team composition, processes and fidelity to the ACT model were gathered from team managers or senior clinicians. All staff completed self-report questionnaires on their work experiences and case managers completed structured data sheets on the characteristics of their patients, as described below. Because of the nature of their work and small case loads, case managers knew their patients very well. They were encouraged to check the case notes for patient data, where necessary.

\section{Measures and Data Collection}

This cross-sectional survey used similar measures to those used in the PLAO studies (Harvey et al., In press). A few items were removed as they were not relevant to the Australian service system. Data gathering was co-ordinated, part-time, by a psychiatrist in training (SM) and by the first author. The study was approved by the relevant Research and Ethics committees.

\section{Team composition, processes and fidelity to the ACT model}

A semi-structured questionnaire was developed specifically for the PLAO studies (Wright et al., 2003) that collects information on team staffing, caseloads, and policies and protocols. An item was added to gather information specific to Victoria regarding the use of CTOs. ACT model fidelity was assessed using the Dartmouth Assertive Community Treatment Scale (DACTS) (Teague et al., 1998). Three sub-scales are generated: human resources; organisational boundaries; and nature of services. Total mean scores above 4 denote high model fidelity, 3 to 4 "ACT-like" services, and below 3, low model fidelity. The DACTS was completed by team leaders after explanation and discussion of items with the third author. Although the DACTS was developed for administration by an evaluator outside the team, Winter \& Calsyn (2000) 
suggest that inter-rater reliability (evaluators and site respondents) is acceptable and that "evaluators appear to have relied heavily on the respondents' ratings of fidelity" (page 331). This provides support for the acceptability of our approach.

\section{Staff experiences}

Job satisfaction was measured using a self-report instrument, the Minnesota Satisfaction Questionnaire, short version (Weiss, Dawis, England, \& Lofquist, 1967). This consists of 20 items rated on a five point scale, producing scores for two subscales, intrinsic and extrinsic satisfaction. A neutral attitude is indicated by scores of 60 for overall satisfaction, 36 for intrinsic satisfaction and 18 for extrinsic satisfaction. Other cutpoints are as follows: a satisfied attitude is indicated by scores of 80 (overall), 48 (intrinsic) and 24 (extrinsic) and a dissatisfied attitude by 40 (overall), 24 (intrinsic) and 12 (extrinsic).

Sources of job satisfaction \& stress were identified using a modified version of a questionnaire by Prosser et al (Prosser et al., 1997) with additional items relevant to ACT work. Sources of job satisfaction were rated on a five point scale where 0 indicated no satisfaction and 4 a very important source of satisfaction. Sources of stress were also rated on a five point scale with 0 indicating not a source of stress and 4 being extremely stressful.

Clinicians were asked to describe and rate (1=very inadequate; $2=$ somewhat inadequate; $3=$ satisfactory) how much training they had received in aspects of the work.

Staff burnout was assessed using the Maslach Burnout Inventory (Maslach, Jackson, \& Leiter, 1996), a 22 item scale that yields scores for three components of burnout: emotional exhaustion; depersonalisation; personal accomplishment. High burnout is reflected by high scores for the first two and a low score for the third component. Amongst mental health professionals, high burnout is characterised by scores greater than 20, greater than 7, and less than 29 respectively (medium burnout: 14-20, 5-7 and 29-33; low burnout: 13 or less, 4 or less, and 34 or more).

\section{Patient characteristics}

Patient socio-demographic details, clinical information and details of adverse events were gathered on 
structured datasheets by case managers. Staff made ratings of their patients' substance use using the Clinician Alcohol and Drug Scales (Drake, Mueser, \& McHugo, 1986) which give ratings from 1 (abstinent) to 5 (dependent with institutionalisation). Use was rated over the previous 6 months.

\section{Data Analysis}

Descriptive data were calculated for all four MSTTs and for each team using SPSS version 19 (SPSS, 2009). Mean scores were calculated for normally distributed continuous variables and one-way ANOVA was carried out for comparisons of means. The Tukey HSD test was used for post hoc comparisons. Medians were calculated for non-normally distributed data and compared using Kruskal-Wallis ANOVA. A Bonferroni correction.was applied in order to take account of multiple comparisons and the significance level was set at $<0.002$.

In order to examine associations between burnout and work satisfaction and stress, factors previously noted to be associated with burnout, such as collaboration with peers, team approach, leadership, bureaucratic constraints and time spent in current post and within mental health, were identified from the literature (Billings et al, 2003; Corrigan et al, 2001 and Rosen et al, 2007). Ten variables that equated to these factors were identified within the questionnaires on staff experiences. These were: supervisor competence; co-workers getting along together; satisfaction with company of other staff; satisfaction with taking a team rather than individual approach with clients; not feeling supported or supervised by seniors; poor communication between colleagues; stress due to taking a team rather than individual approach with clients; stress due to insufficient resources; feeling responsibility without power; too much administration. Their distribution was examined. The variable 'stress due to taking a team rather than individual approach with clients' was treated as dichotomous (presence or absence of stress due to taking a team approach) since the skewness / standard error (SE) of skewness ratio was 3.40 with 29 clinicians scoring 0 and 15 scoring 1 or more. Correlations were performed between the three components of burnout (emotional exhaustion, depersonalisation and personal accomplishment) and the afore-mentioned ten clinician

variables. Spearman correlations were performed for dichotomous or skewed variables and Pearson correlations for variables that were not skewed. A Bonferroni correction was applied with an adjusted twotailed probability of $\mathrm{p}<0.002$. 


\section{Results}

\section{Response rates}

All four team questionnaires were completed (100\%). The response rate for the staff questionnaires was 98\% (44 of 45). Data were collected on 183 of a possible 202 patients (91\%).

\section{Team operation and composition}

All teams had been in operation between six and nine years, were aligned with geographical areas (catchment areas) and were similar in size. The mean clinical Equivalent Full Time (EFT) positions, excluding psychiatrists, were 8.0 (range $=7.1-9.0$ ). Psychiatric nursing was the most common profession (mean 5.1 EFT, range = 3.0-7.0). Social workers (mean 1.4 EFT, range $=1.0-2.0)$ and occupational therapists (mean 1.5 EFT, range $=1.0-3.0$ ) were employed across teams but there were no psychologists. All teams had psychiatric input, with either 0.3 or 0.4 consultant EFT per team, and 0.5 to 0.8 EFT medical officers or psychiatric trainees.

\section{Referrals, eligibility criteria and caseloads}

All four MSTTs worked with patients aged between 18 and 65 years. Most referrals were accepted from CMHTs (or continuing care teams), specialist mental health services such as forensic mental health and primary care. All teams had written eligibility criteria specifying that patients with multiple admissions were accepted and, except for team 3, patients who were difficult to engage with other services. None of the teams had written exclusion criteria. Team 4 targeted young people and those with comorbid substance use. All had designated maximum caseloads (range $=45$ to 63 ) and limits to caseload per clinical EFT. The mean caseload for the four teams was 51 (range $=45-60)$. The average caseload per EFT was 6.3 (range $=$ 5.6-6.7). The average number of patients discharged in the preceding six months was 11 (range $=9-12$ ).

\section{Patterns of working}

All teams operated outside usual office hours and over weekends. On average, $80 \%$ of contacts with patients occurred in the community (outside community mental health settings). Although none of the teams had designated inpatient beds available to them, all had some input into the management of their 
patients on the ward.

\section{Model fidelity}

The mean DACTS score for all four teams was 3.7 ( $\mathrm{SD}=0.3$, range $=3.4-4.0)$, scoring as "ACT-like". 13 of 28 DACTS items had a mean score above 4 ('high fidelity'), 10 items had means of 3-4 ('moderate fidelity'), and 5 had a mean score of below 3 ('low fidelity'). Four of the 5 'low fidelity' items concerned whether staff with specific expertise were employed within the teams, namely substance abuse specialists, vocational workers or consumers.

\section{Staff socio-demographic and job details}

Most clinicians were women (59\%), and aged between 36 and 54 years (52\%). Half identified as either British or Irish, with about one third Australian. Clinicians had worked a median of 1.5 years (range $=1$ month to 8 years) in their current team and, on average, 3.4 years $(\mathrm{SD}=3.2$ years, range $=1$ month to 11.3 years) in MSTTs and 10.4 years $(\mathrm{SD}=6.2$ years, range $=8$ months to 25 years) in other mental health programs.

\section{Job satisfaction and stress}

Clinicians were fairly satisfied with their work with ratings falling just short of the satisfied cutpoint (mean Minnesota Satisfaction Scale [25] for all four teams: general satisfaction 76.7 [SD $=8.5$, range $=53$ 97]; intrinsic satisfaction 45.9 [ $\mathrm{SD}=5.7$, range $=30-57]$; extrinsic satisfaction $22.8[\mathrm{SD}=2.8$, range $=16$ 30]). The most important sources of satisfaction (Prosser et al., 1997) were: spending time with patients helping them cope with their difficulties; working intensively with a small number of patients; working in a new type of team that is intended to improve standards of care (all scored 3 as important). No items were rated as moderately stressful (or worse) across all MSTTs (Prosser et al., 1997). The three most stressful issues, rated between mildly and moderately stressful (scores of 1 and 2, respectively), were: the risk of patients harming self or others; not enough people or resources to do the job properly; and aggressive behaviour towards staff on the part of patients.

\section{Burnout}

The average burnout scores (Maslach et al., 1996) for all four teams were medium for emotional 
exhaustion (mean 16.5, $\mathrm{SD}=8.7$, range $=2-37$ ) and low for depersonalisation (median 4.0, range $=0-17$ ) and personal accomplishment (mean 35.8, $\mathrm{SD}=7.3$, range $=12-47$ ).

Correlations between the ten clinician experience variables and the three components of burnout: emotional exhaustion, depersonalisation and personal accomplishment, were examined. Increased depersonalisation was associated with increased stress due to feelings of responsibility without power $(\mathrm{r}=$ 0.522; $\mathrm{p}<0.001)$, decreased satisfaction with supervisor competence $(\mathrm{r}=-0.538 ; \mathrm{p}<0.001)$ and increased stress due to taking a team rather than an individual approach $($ rho $=0.470 ; \mathrm{p}=0.001)$.

\section{Training}

Just under three quarters of staff felt they had either had all or most of the training they required for their job. The least satisfactory areas of training (all scored between $2=$ somewhat inadequate and $3=$ satisfactory) were: providing advice regarding benefits entitlements, housing and other social problems; working with dual diagnosis patients; working with patient's families; working with patients to increase willingness to take medication; and helping patients to cope with distressing symptoms such as hallucinations and paranoid thoughts.

\section{Characteristics of patients receiving ACT}

Most patients receiving ACT were single (72.2\%), male (63.0\%) and of Australian origin (62.2\%). Their mean age was 37.4 years $(\mathrm{SD}=11.4$, range $=18-67$ years). A minority were employed $(8.3 \%)$ or studying (2.8\%). The majority (79.6\%) were living in independent accommodation, with most either living alone $(45.3 \%)$ or with their parents $(24.9 \%)$. One in six had lived in marginal accommodation and just over one in ten had a history of homelessness ( 5 days or more) in the previous two years. For these individuals, the average time spent in these settings was 63 days (range $=3-730$ ) and 30 days (range $=5-700$ ), respectively.

Three quarters of MSTT patients had a diagnosis of schizophrenia and atypical antipsychotics were the most commonly prescribed treatment (60.6\%). Patients had experienced a median of 7.5 inpatient admissions in total (range $=0-80$ ), with two inpatient episodes (range $=0-11$ ) of median duration of 40 days (range $=0-456$ days) within the previous two years. Four in five had experienced an involuntary 
admission in the preceding two years with just over half (55.5\%) currently treated under a CTO. Over fourteen per cent currently experienced alcohol abuse or dependence and substance abuse or dependence were experienced by $24.0 \%$. Just over one third (35.8\%) had been violent in the previous two years, with most of these episodes (60.9\%) requiring police intervention. Fewer (16.8\%) had experienced an episode of self harm in the same period.

\section{Differences between the teams}

\section{Team characteristics}

Team 1 had only moved to extended hours of operation in the previous year. Team 3 was the only team that did not target patients who were difficult to engage with other services. Further, most of this team's clinicians were allied health professionals, whereas the other teams had predominantly nursing staff. Although the overall rate of contact with patients in the community was high, team 3 had the lowest rate $(65-79 \%)$.

\section{Staff characteristics}

Team 3 had the youngest staff: most were less than 35 years old with less than ten years experience in mental health. Several of the professional groups in team 3 had most of their experience in ACT. In contrast, most staff in team 1 had greater experience in other mental health programs and less experience in ACT compared with other teams. Most staff in teams 1 and 3 (82\% and 70\%, respectively) had been recently recruited (within the last two years). There was less staff turnover in teams 2 and 4 (42\% and 55\%, respectively).

Although sources of satisfaction for all clinicians were similar, there were potentially important and significant differences between each team with respect to overall job satisfaction and the subscale components (see Table 1). In particular, team 1 clinicians were consistently less satisfied than clinicians from team 3 for intrinsic, extrinsic and general satisfaction (one-way ANOVA $F(3,39)=8.35$, p<0.0001, $\mathrm{F}(3,40)=7.48, \mathrm{p}<0.0001, \mathrm{~F}(3,39)=8.88, \mathrm{p}<0.0001$, respectively $)$. There were also significant differences in job satisfaction between team 1 and teams 2 and 4 (see Table 1 for details). In all cases, the Tukey HSD test found these differences were significant at the $\mathrm{p}<0.05$ level. Patient-related factors were prominent 
sources of stress for all teams. However, team 1 was the only team citing lack of support and supervision from seniors as among the four most stressful items.

Table 1 about here

Differences in burnout scores between teams did not reach statistical significance using the stringent significance level set at $<0.002$ although differences in emotional exhaustion almost reached this level. Team 1 was the only team to score highly on emotional exhaustion. Further, team 3 had consistently lower burnout than any other team.

\section{Patient characteristics}

Socio-demographic characteristics of patients were very similar between teams, apart from the proportion living alone $\left(\chi^{2}=21.4, \mathrm{p}<0.001\right)$, with a significantly higher rate of patients living alone in team $1(72 \%)$ compared with team $4(23 \%)$.

Teams 1 and 4 had fewer patients with a diagnosis of schizophrenia (61\% and 65\%, respectively) and higher rates of prescription of mood stabilisers (55\% and 39\%, respectively) than other teams. Rates of depot prescription did not vary much between teams but atypical anti-psychotic prescription was highest in team $2(77 \%)$ and lowest in team $1(40 \%)$. Substance abuse and dependency during the previous 6 months were higher in teams 1 and 2. Admission histories, including days spent in hospital over the previous two years, were similar. The proportion of patients on CTOs varied between 39\% (team 4) and 63\% (team 2).

Table 2 about here

\section{Discussion}

This is the first description of the structure and processes of Australian ACT teams, including staff and patient characteristics. Strengths of the study include a very high response rate and the employment of previously validated questionnaires used in the PLAO studies (Billings et al., 2003; Priebe et al., 2003; Wright et al., 2003). Case managers completed questionnaires gathering patient data in this study (rather than research assistants in the PLAO studies) for pragmatic resource reasons. However as reported 
elsewhere, this does not appear to have compromised the quality of the data (Harvey et al., In press). A limitation of this study is that it was not designed to collect outcome data and cannot make any interpretations concerning effectiveness of ACT in this setting. Also, the ACT teams were well-established and selected from relatively uniform catchment areas in metropolitan Melbourne. Therefore it is unclear whether these findings are generalisable elsewhere in Victoria or Australia.

All teams were ACT-like and operating quite closely to ACT model specifications, with one team just short of high model fidelity with a score of 4.0. Low fidelity aspects of the model were common to all teams. These included specialists (consumer consultants, substance abuse and vocational specialists) who were not located within the teams, although all were employed elsewhere within the broader mental health or service system. The lack of vocational specialists may explain the low rates of employment amongst these ACT patients despite evidence that this model can be effective in increasing employment rates (Bond, Drake, Mueser, \& Becker, 1997), especially if these specialists are integrated with clinical mental health services. In Victoria, vocational specialists are not typically well integrated since they are employed within agencies funded by the federal government and in some state-funded non-clinical rehabilitation services. These findings may support the need for greater integration of these statutory and non-statutory services with those in clinical mental health (Kirsh, Cockburn, \& Gewurtz, 2005). Since the survey data were gathered, nurses with specialist skills in substance misuse have been appointed to Victorian MSTTs. Further, MSTT clinicians recognised a need for increased training for themselves in this area. The team that focussed on this group had patients with low ratings for substance misuse over the last six months which may suggest that its approach was successful.

The four MSTTs were broadly similar in size, composition and operation. Team 3 was the most distinct being the only team that did not specifically target patients who were difficult to engage and also had the lowest proportion of contacts with patients at home; these observations are likely to be linked. Since home visiting appears to be an "active component" of the ACT model (Catty et al., 2002; Harvey et al., In press), it must be implemented and sustained. Unlike the other teams, team 3 was composed mostly of allied health professionals who were younger, less experienced in mental health work and recently recruited. It is plausible that greater staff experience of mental health services and/or ACT may be needed to enable a team to work effectively with the target population in their homes. This is consistent with the observation 
that ACT staff use more varied and flexible strategies than staff in CMHTs to engage patients (Killaspy et al., 2009). Further, there are likely to be inter-disciplinary differences in confidence with assertive engagement techniques which may be reflected in these findings, perhaps providing additional support for multidisciplinary team working as an important component of successful ACT (Burns et al., 2007; Rosen, Mueser, \& Teesson, 2007). These differences in team operation may also help explain the consistently low burnout of team 3 clinicians.

A telling finding for the sustainability of the ACT model was that clinicians were fairly satisfied overall with intrinsic aspects of the work (Killaspy et al., 2009). Further, Australian ACT clinicians' satisfaction ratings were slightly more positive than London clinicians (Billings et al, 2003). Unlike the PLAO studies, we did not identify associations between burnout and either time spent in current post or within mental health. No aspects of the work were rated as moderately stressful (or worse). Further, staff burnout was either rated as medium or low. Nonetheless, these overall positive findings obscure some potentially important differences between teams. Team 1 clinicians were least satisfied and most stressed by their work compared with other teams and were the only group experiencing high emotional exhaustion. Most team 1 clinicians had recently joined the team, had the least experience in ACT and had only recently moved to extended hours of operation, all factors that might account for their higher stress. They were also the only team to report lack of support and supervision from seniors as among the most stressful items. This is in keeping with a reported association between burnout and diminished collegial support and the observation that uninvolved leaders undermine teamwork (Corrigan, Steiner, McCracken, Blaser, \& Barr, 2001). The correlational analyses provide further support for this interpretation. Our finding that increased burnout was also associated with greater stress due to taking a team rather than an individual approach highlights the need for better understanding of what constitutes effective team working and clinical leadership when treatment is delivered through a team-based approach.

The typical MSTT patient is a single man with a diagnosis of schizophrenia, living in independent accommodation and with frequent previous admissions to hospital. This latter characteristic suggests that Melbourne ACT teams are targeting the relevant population. A significant minority of MSTT patients had a history of unstable housing and episodes of violence were quite frequent, a high proportion involving the police. This reflects a patient group that is marginalised and relatively disengaged from services, indicating 
a need not only for clinical interventions but also for ongoing service coordination involving housing, police and other community agencies.

There were few significant differences between the patients of each team although team 1 patients were less likely to have a diagnosis of schizophrenia, more likely to be prescribed a mood stabiliser and less likely to be prescribed atypical antipsychotics than patients of other teams. Rates of substance use were high among team 1 (and team 2) patients and they were more likely to be living alone. These findings may reflect underlying differences between the catchment areas and could indicate a client group more difficult to treat within the ACT model which may also explain the satisfaction, stress and burnout findings for team 1 clinicians.

It has been argued that the organisation of service delivery in line with ACT specifications is only a vehicle for the delivery of treatment of adequate content and quality (Burns et al., 1999; Issakidis et al., 1999; Rosen et al., 2007). In this regard, MSTT clinicians' accounts of their training needs were almost identical to those of London ACT clinicians (Billings et al., 2003) and equate to recognition of a relative lack of confidence and skill in delivering psychosocial interventions. The absence of psychologists within Melbourne teams may add to this problem. Specific training of ACT clinicians in assertive engagement techniques and effective multidisciplinary team working are also suggested by the present findings. Treatment content and unique contextual factors (such as the use of CTOs in Victoria) as well as subtle but discernible variations between ACT teams require further study (Billings et al., 2003), especially with respect to their contribution to the operation and effectiveness of the model. In particular, it is plausible that the ready availability of CTOs may both contribute to enhanced model fidelity and clinicians' feelings that they can work in this model. Finally, although it is not possible to draw conclusions about effectiveness, this study provides evidence that within a relatively well-resourced mental health system with a clearlyspecified service framework, it is possible to implement an evidence-based model and achieve acceptable model fidelity when assessed some years later.

\section{Acknowledgments}

The authors thank all the MSTT team leaders and clinicians who participated in this study. 


\section{References}

Ash, D., Brown, P., Burvill, P., Davies, J., Hughson, B., Meadows, G., et al. (2007). Mental health services in the Australian states and territories. In G. Meadows, B. Singh \& M. Grigg (Eds.), Mental health in Australia: Collaborative community practice. (2nd ed., pp. 99-131). South Melbourne: Oxford University Press.

Australian Bureau of Statistics. (2003a). Regional population growth Australia and New Zealand, 2001 2002 (Publication. Retrieved 10th July 2007: http://www.abs.gov.au/AUSSTATS/abs@.nsf/ProductsbyReleaseDate/29D57E46C7CB3897CA2 56E54007167F3?OpenDocument\#

Australian Bureau of Statistics. (2003b). 2001 Census: Socio-economic indices for areas. Canberra: Australian Bureau of Statistics.

Billings, J., Johnson, S., Bebbington, P., Greaves, A., Priebe, S., Muijen, M., et al. (2003). Assertive outreach teams in London: Staff experiences and perceptions. Pan-London Assertive Outreach Study, part 2. British Journal of Psychiatry, 183, 139-147.

Bond, G. R. (1990). Intensive case management. Hospital and Community Psychiatry, 41(8), 927-928.

Bond, G., Drake, R. E., Mueser, K. T., \& Becker, D. R. (1997). An update on supported employment for people with severe mental illness. Psychiatric Services, 48(3), 335-346.

Burns, T., Catty, J., Dash, M., Roberts, C., Lockwood, A., \& Marshall, M. (2007). Use of intensive case management to reduce time in hospital in people with severe mental illness: Systematic review and meta-regression. British Medical Journal, 335(7615), 336-342.

Burns, T., Creed, F., Fahy, T., Thompson, S., Tryrer, P., \& White, I. (1999). Intensive versus standard case management for severe psychotic illness: A randomised trial. Lancet, 353(9171), 2185-2189.

Catty, J., Burns, T., Knapp, M., Watt, H., Wright, C., Henderson, J., et al. (2002). Home treatment for mental health problems: A systematic review. Psychological Medicine, 32, 383-401.

Corrigan, P. W., Steiner, L., McCracken, S. G., Blaser, B., \& Barr, M. (2001). Strategies for disseminating evidence-based practices to staff who treat people with serious mental illness. Psychiatric Services, 52(12), 1598-1606.

Drake, R., Mueser, K., \& McHugo, G. (1986). Clinician ratings scales: Alcohol Use Scale (AUS), Drug Use Scale (DUS) and Substance Abuse Treatment Scale (SATS). In L. Sederer \& B. Dickey (Eds.), Outcomes assessment in clinical practice. Baltimore: Williams and Wilkins.

Fiander, M., Burns, T., McHugo, G. J., \& Drake, R. E. (2003). Assertive community treatment across the Atlantic: Comparison of model fidelity in the UK and USA. British Journal of Psychiatry, 182, 248-254.

Hambridge, J. A., \& Rosen, A. (1994). Assertive community treatment for the seriously mentally ill in suburban Sydney: A programme description and evaluation. Australian and New Zealand Journal of Psychiatry, 28, 438-445.

Harvey, C., Killaspy, H., Martino, S., White, S., Priebe, S., Wright, C., et al. (In press). A comparison of the implementation of Assertive Community Treatment in Melbourne, Australia and London, England. In press Epidemiologia Psichiatria Sociale.

Health and Community Services. (1994). Victoria's Mental Health Service. The framework for service delivery. Melbourne: Psychiatric Services division, Victoria.

Hoult, J. (1986). Community care of the acutely mentally ill. British Journal of Psychiatry, 149, $137-144$.

Human Services Victoria. (1995). Mobile support and treatment services. Guidelines for service provision. Melbourne: Psychiatric Services Branch.

Issakidis, C., Sanderson, K., Teeson, M., Johnson, S., \& Buhrich, N. (1999). Intensive case management in Australia: A randomized controlled trial. Acta Psychiatrica Scandinavica, 99, 360-367.

Joy, C. B., Adams, C. E., \& Rice, K. (2002). Crisis intervention for people with severe mental illness (Cochrane Review). In The Cochrane Library. Oxford: Update Software.

Killaspy, H., Bebbington, P., Blizard, R., Johnson, S., Nolan, F., Pilling, S., et al. (2006). The REACT study: Randomised evaluation of assertive community treatment in north London. British Medical Journal, 332(7545), 815-820.

Killaspy, H., Johnson, S., Pierce, B., Bebbington, P., Pilling, S., Nolan, F., et al. (2009). Successful engagement: a mixed methods study of the approaches of assertive community treatment and community mental health teams in the REACT trial. Social Psychiatry and Psychiatric Epidemiology, 44(7), 532-540.

King, R. (2006). Intensive case management: a critical re-appraisal of the scientific evidence for effectiveness. Administration Policy Mental Health and Mental Heath Services Research, 33(5), 529-535. 
Kirsh, B., Cockburn, L., \& Gewurtz, R. (2005). Best practice in occupational therapy: Program characteristics that influence vocational outcomes for people with serious mental illnesses. Canadian Journal of Occupational Therapy, 72(5), 265-279.

Marshall, M., Bond, G., Stein, L. I., Shepherd, G., McGrew, J., Hoult, J., et al. (1999). PRiSM psychosis study. Design limitations, questionable conclusions. British Journal of Psychiatry, 175, 501-503.

Marshall, M., \& Lockwood, A. (1998). Assertive community treatment for people with severe mental disorders (Review) (Publication no. CD001089. DOI: 10.1002/14651858). Retrieved 4th November 2005, from John Wiley \& Sons Ltd:

Maslach, C., Jackson, S. E., \& Leiter, M. P. (1996). The Maslach Burnout Inventory (3rd ed.). Palo Alto, CA: Consulting Psychologists Press.

McGrew, J. H., Bond, G. R., Dietzen, L., \& Salyers, M. (1994). Measuring the Fidelity of Implementation of a Mental Health Program Model. Journal of Consulting and Clinical Psychology, 62(4), 670678.

McHugo, G. J., Drake, R. E., Whitley, R., Bond, G. R., Campbell, K., Rapp, C. A., et al. (2007). Fidelity outcomes in the National Implementing Evidence-Based Practices Project. Psychiatric Services, 58(10), 1279-1284.

Priebe, S., Fakhoury, W., Watts, J., Bebbington, P., Burns, T., Johnson, S., et al. (2003). Assertive outreach teams in London: Patient characteristics and outcomes. Pan-London Assertive Outreach Study, part 3. British Journal of Psychiatry, 183, 148-154.

Prosser, D., Johnson, S., Kuipers, E., Szmukler, G., Bebbington, P., \& Thornicroft, G. (1997). Perceived sources of work stress and satisfaction among hospital and community mental health staff, and their relation to mental health, burnout and job satisfaction. Journal of Psychosom Research, 43(1), 51-59.

Rosen, A., Mueser, K., \& Teesson, M. (2007). Assertive community treatment-Issues from scientific and clinical literature with implications for practice. Journal of Rehabilitation Research and Development, 44(6), 813-825.

Salyers, M. P., Bond, G. R., Teague, G. B., Cox, J. F., Smith, M. E., Hicks, M. L., et al. (2003). Is it ACT yet? Real-world examples of evaluating the degree of implementation for assertive community treatment. Journal of Behavioral Health Services and Research, 30(3), 304-320.

SPSS. (2009). SPSS for Windows, Version 19. Chicago: SPSS Inc.

Stein, L. I., \& Test, M. A. (1980). Alternatives to mental hospital treatment. Archives of General Psychiatry, 37, 392 - 397.

Sytema, S., Wunderink, L., Bloemers, W., Roorda, L., \& Wiersma, D. (2007). Assertive community treatment in the Netherlands: a randomized controlled trial. Acta Psychiatrica Scandinavica, 116(2), 105-112.

Teague, G., Bond, G., \& Drake, R. (1998). Program fidelity in assertive community treatment: Development and use of a measure. American Journal of Orthopsychiatry, 68(2), 216-232.

Thornicroft, G., Wykes, T., Holloway, F., Johnson, S., \& Szmukler, G. (1998). From efficacy to effectiveness in community mental health services. PRiSM Psychosis Study 10. British Journal of Psychiatry, 173, 423-427.

Victorian Government. (1986). Mental Health Act 1986. Melbourne: Department of Human Services.

Weiss, D. J., Dawis, R. V., England, G. W., \& Lofquist, L. H. (1967). Manual for the Minnesota Satisfaction Questionnaire. Minnesota Studies in Vocational Rehabilitation: XXII. Minneapolis: University of Minnesota, Industrial Relations Center Work Adjustment Project.

Wright, C., Burns, T., James, P., Billings, J., Johnson, S., Muijen, M., et al. (2003). Assertive outreach teams in London: Models of operation. Pan-London Assertive Outreach Study, part 1. British Journal of Psychiatry, 183, 132-138.

Table 1:

$\underline{\text { Mean (and SD) scores for staff satisfaction for each team: Minnesota Satisfaction Scale }}$ 
${ }^{a}$ Variances not significantly different, Levene's Test $F=0.83, p=0.49$, Tukey HSD test: team 1 significantly differed from teams 2,3 and $4(\mathrm{p}<0.05)$

${ }^{b}$ Variances not significantly different, Levene's Test $\mathrm{F}=1.20, \mathrm{p}=0.32$, Tukey HSD test: team 1

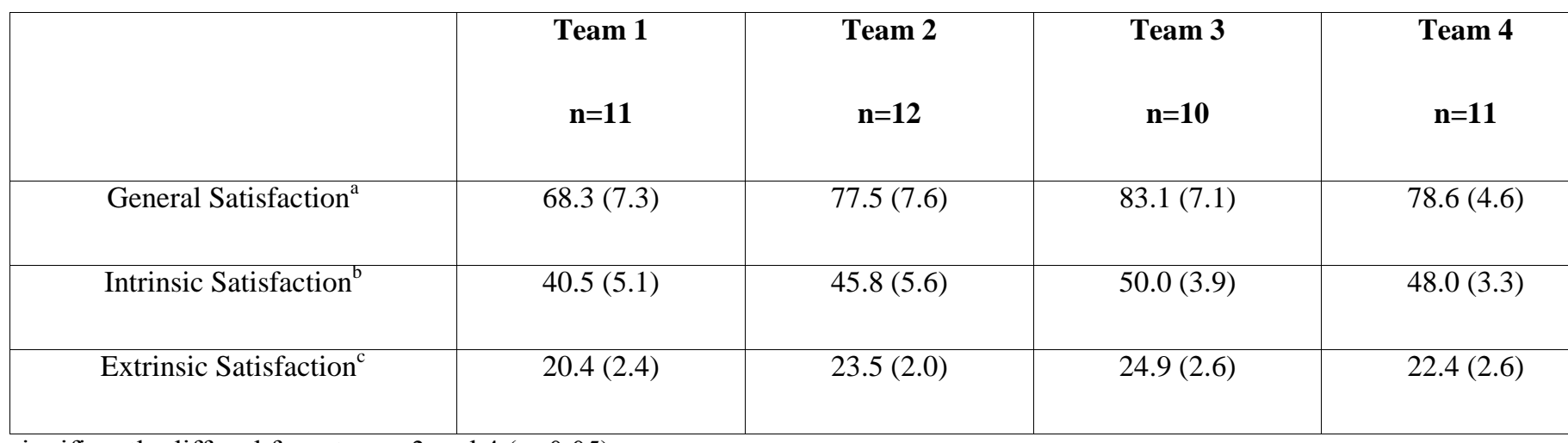

significantly differed from teams 3 and $4(\mathrm{p}<0.05)$

${ }^{\mathrm{c}}$ Variances not significantly different, Levene's Test $\mathrm{F}=0.21, \mathrm{p}=0.89$, Tukey HSD test: team 1

significantly differed from teams 2 and $3(\mathrm{p}<0.05)$

Table 2:

Clinical characteristics of patients by team 


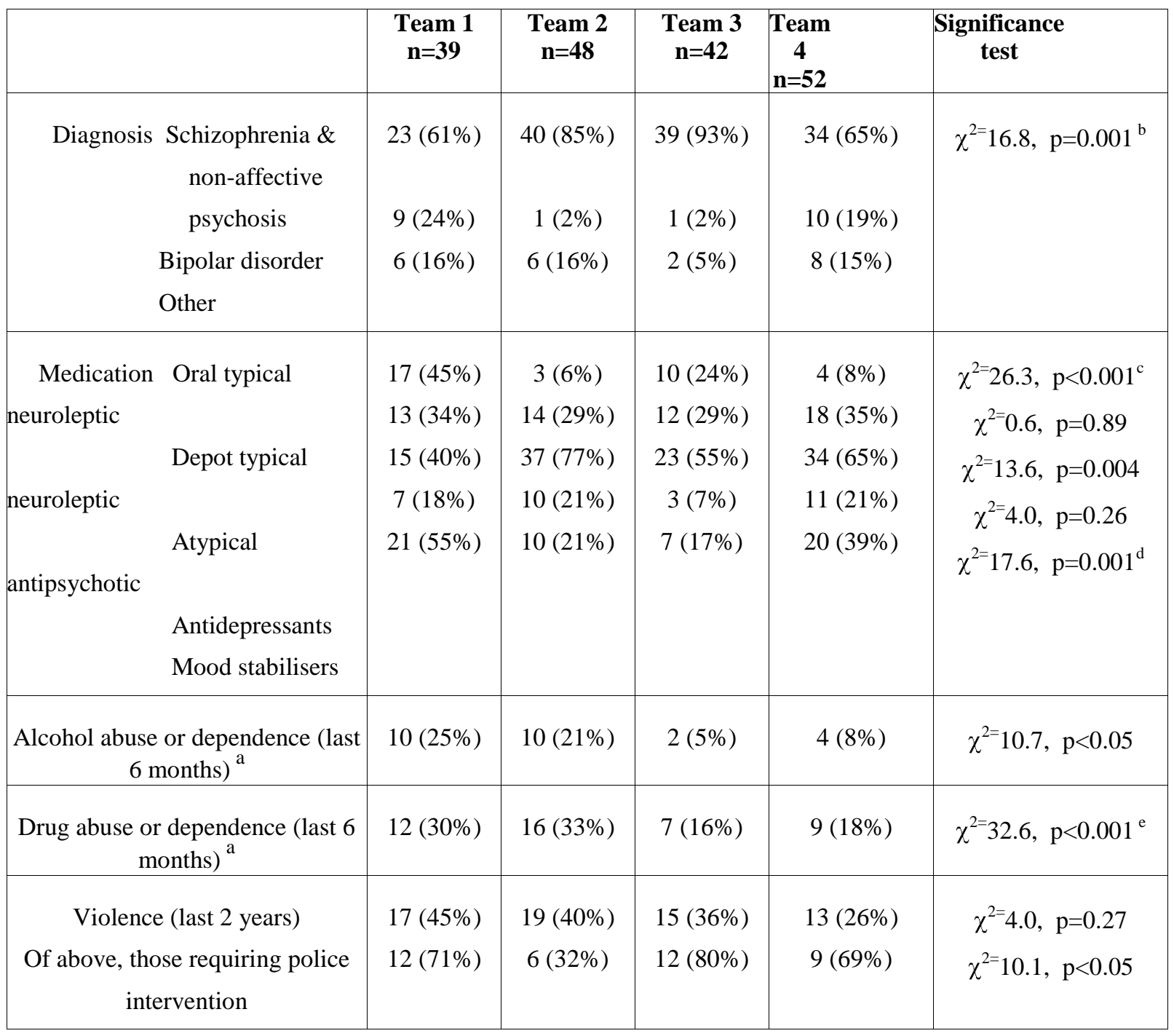

\begin{tabular}{|c|c|c|c|}
\hline Deliberate self harm (last 2 years) & $7(18 \%)$ & $6(13 \%)$ & $4(10 \%)$ \\
\hline $\begin{array}{c}\text { Street homelessness (last 2 years) \%caseload } \\
\text { mean days }\end{array}$ & $\begin{array}{c}23 \% \\
52 \text { days }\end{array}$ & $8 \%$ \\
60 days & 318 days \\
\hline $\begin{array}{c}\text { Marginal accom (last 2 years) } \\
\text { mean, median days }\end{array}$ & $31 \%$ & $10 \%$ & $14 \%$ \\
Total admissions (mean, median) & 154,68 days & 97,30 days & 118,80 days \\
\hline
\end{tabular}




\begin{tabular}{|c|c|c|}
\hline Admissions during last 2 years (mean, median) & 3,3 & 2,2 \\
\hline Days in hospital during last 2 years (median) & 49 & 30 \\
\hline Involuntary admissions to hospital during last 2 years & $32(87 \%)$ & $39(81 \%)$ \\
\hline
\end{tabular}

${ }^{a}$ Items scored as either 3 or more indicating abuse, dependence or severe dependence.

${ }^{\mathrm{b}}$ significant difference between teams 1 and 3

${ }^{\mathrm{c}}$ significant differences between teams 1 and $2 \& 1$ and 4

d significant differences between teams 1 and $2 \& 1$ and 3

e significant differences between teams 4 and $1 \& 4$ and 2 